\title{
Le massif forestier, objet pertinent pour la recherche archéologique. L'exemple du massif forestier de Haye (Meurthe-et-Moselle)
}

Forest massif, a pertinent object for archaeological research. The example of the Haye forest (Meurthe-et-Moselle)

Der Waldkomplex, ein sachdienliches Studienobjekt für die archäologische Forschung. Das Beispiel des Waldes von Haye (Meurthe-et-Moselle)

Murielle Georges-Leroy, Jérôme Bock, Etienne Dambrine et Jean-Luc Dupouey

\section{OpenEdition}

Journals

Édition électronique

URL : http://journals.openedition.org/rge/1931

DOI : $10.4000 /$ rge. 1931

ISSN : $2108-6478$

Éditeur

Association des géographes de l'Est

Édition imprimée

Date de publication : 1 janvier 2009

ISSN : 0035-3213

\section{Référence électronique}

Murielle Georges-Leroy, Jérôme Bock, Etienne Dambrine et Jean-Luc Dupouey, « Le massif forestier, objet pertinent pour la recherche archéologique. L'exemple du massif forestier de Haye (Meurthe-etMoselle) », Revue Géographique de l'Est [En ligne], vol. 49 / 2-3 | 2009, mis en ligne le 01 avril 2013, consulté le 08 septembre 2020. URL : http://journals.openedition.org/rge/1931 ; DOI : https://doi.org/ 10.4000/rge.1931

Ce document a été généré automatiquement le 8 septembre 2020

Tous droits réservés 


\section{Le massif forestier, objet pertinent pour la recherche archéologique. L'exemple du massif forestier de Haye (Meurthe-et-Moselle)}

Forest massif, a pertinent object for archaeological research. The example of the Haye forest (Meurthe-et-Moselle)

Der Waldkomplex, ein sachdienliches Studienobjekt für die archäologische Forschung. Das Beispiel des Waldes von Haye (Meurthe-et-Moselle)

Murielle Georges-Leroy, Jérôme Bock, Etienne Dambrine et Jean-Luc Dupouey

Les forêts recèlent de nombreux vestiges archéologiques, souvent conservés sous forme de micro-reliefs plus ou moins prononcés. La forêt a un caractère protecteur: les vestiges y sont en général beaucoup moins érodés ou perturbés que dans les zones agricoles ou urbanisées. Pourtant, du fait de contraintes dans leur repérage, les vestiges conservés en forêt ne sont pas toujours étudiés par les archéologues et l'absence de sites dans certains massifs forestiers est souvent le résultat d'un manque de prospection archéologique plutôt que d'une absence réelle. En effet, en matière de prospection deux approches, très souvent exclusives, existent chez les archéologues. Il y a d'un côté ceux, les plus nombreux, qui cherchent des sites en milieu labouré et de l'autre ceux qui concentrent leurs recherches en forêt. Les éléments observables sont en effet de nature très différente. En milieu labouré les sites se présentent sous la forme d'épandages plus ou moins étendus d'artefacts (objets, éléments de construction, etc.), alors qu'en forêt ils apparaissent sous forme de micro-reliefs voire d'anomalies de végétation (Ferdière 1998). Ces anomalies témoignent alors d'un impact des occupations anciennes sur la biodiversité actuelle. Les préoccupations de l'archéologue, du forestier et de l'écologue se rejoignent donc. 
Nous souhaitons illustrer ces convergences et montrer en quoi le massif forestier est un objet pertinent pour la recherche archéologique, en nous appuyant sur le programme interdisciplinaire en cours sur le massif forestier de Haye, situé à côté de Nancy, dans le département de la Meurthe-et-Moselle. Ce programme regroupe des archéologues du Service Régional de l'Archéologie de Lorraine et de l'Institut National de Recherches Archéologiques Préventives, ainsi que des chercheurs du centre de Nancy de l'Institut National de Recherches Agronomiques et de l'office National des Forêts. Mené depuis 1998, il porte sur les habitats et parcellaires fossiles gallo-romains conservés sous couvert forestier sur les plateaux calcaires entre Pont-à-Mousson et Neufchâteau, ainsi que sur leur impact sur la biodiversité et la fertilité actuelles (Dupouey et al. 2002a et 2007b; Georges-Leroy et al. 2003 et 2007).

D'une surface d'environ $11600 \mathrm{ha}$, le massif forestier de Haye est essentiellement constitué de feuillus, hêtraie-chênaie-charmaie principalement. Il a été très durement touché par la tempête Lothar du 26 décembre 1999, puisque près de la moitié des peuplements ont été détruits à plus de $50 \%$. Il est localisé dans une boucle de la Moselle entre Nancy et Toul, en limite sud de la confluence avec la Meurthe (fig. 1). En raison de sa position sur le plateau en bordure de la cuesta des Côtes de Moselle, il est entaillé par de profonds vallons et est limité au nord, à l'est et au sud par de fortes côtes. Géologiquement la majeure partie des terrains se localise sur les calcaires oolithiques du Bajocien, recouverts de placages limoneux-argileux à sableux d'épaisseur et d'étendue variable (BRGM 1978 et 1984). La carte pédologique fait apparaître des sols actuels brunifiés, plus ou moins décarbonatés en surface, qui se développent sur ces formations bajociennes (Gury 1972).

Figure 1 : Le massif forestier de Haye

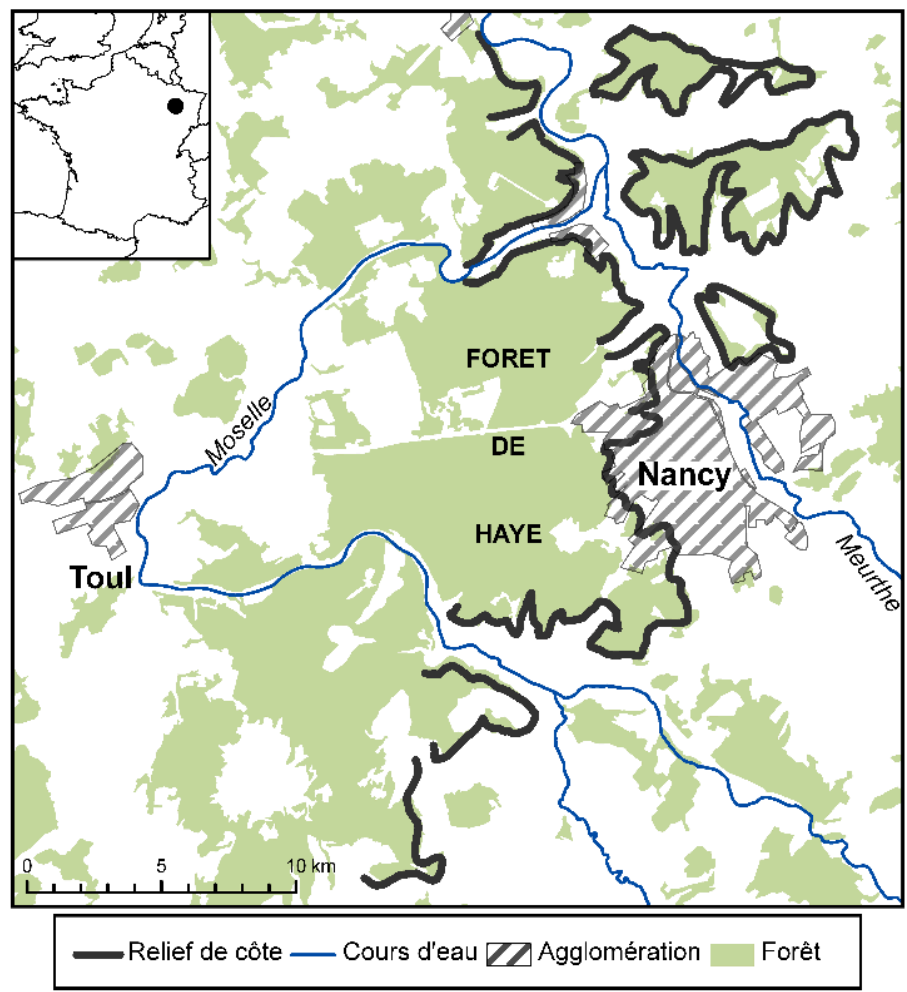




\section{I - La prospection archéologique en milieu forestier}

\section{A - De nombreuses contraintes et limites}

4 En forêt, les vestiges archéologiques visibles en prospection au sol se trahissent soit par des micro-reliefs soit par des indices phytologiques. Seule une partie des vestiges est accessible par cette approche. Ainsi, on repère aisément des nécropoles tumulaires protohistoriques, mais pas les tombes simplement creusées dans le sol, ou encore des villas gallo-romaines en pierre, mais pas les bâtiments construits en matériaux périssables (avec poteaux en bois et torchis). En revanche, les structures agraires et parcellaires anciennes, qui sont souvent totalement érodées et démantelées en milieu labouré, peuvent au contraire avoir été "fossilisées " sous le couvert forestier. Le repérage des sites doit toutefois se faire l'hiver, lors du repos végétatif, pour des questions de visibilité.

5 Un autre handicap de la prospection en forêt réside souvent dans la difficulté à dater les vestiges repérés, car le mobilier archéologique est enfoui dans le sol et ne peut être atteint que lors de sondages ou de fouilles. Certaines circonstances particulières permettent parfois de lever cette contrainte, c'est le cas lorsque des arbres sont abattus par des vents violents et que les chablis remontent du mobilier archéologique, tout comme les labours. Si les tempêtes détruisent partiellement des sites, elles sont donc l'occasion d'une meilleure connaissance de ces sites. Des opérations de fouille systématique de chablis ont ainsi été menées suite à la tempête du 26 décembre 1999 (par exemple Deru 2003). De même, en forêt de Haye, tous les chablis localisés sur des habitats antiques ont été sondés lors des prospections ce qui a permis leur datation.

6 Une fois les sites détectés, il convient de les cartographier, étape indispensable à leur analyse. Là encore un certain nombre de contraintes existent. Les méthodes de topographie classique au tachéomètre sont ralenties par la présence des arbres et parfois inutilisables, tandis que le GPS à correction différentielle a une précision altérée sous couvert forestier, mais offre toutefois une grande souplesse d'utilisation. Par ailleurs, les méthodes de prospection géophysique (électrique, électromagnétique, radar, etc.) qui permettent d'explorer le sous-sol de manière non destructive sont également difficiles à mettre en œuvre en forêt du fait de la présence des arbres et de la végétation basse.

7 Enfin, certains secteurs forestiers sont quasiment inaccessibles au sol, notamment les zones de régénération dense et surtout les zones détruites par des tempêtes depuis quelques années, qui ont été recolonisées par une végétation basse et des ronces et sont encombrées de bois morts et de chablis. Or la prospection aérienne, pourtant très utilisée en archéologie pour la détection de sites, n'est utilisable en milieu forestier que dans de rares conditions. Une voie antique a ainsi été repérée en 2000 par Michel Loiseau dans de jeunes peuplements en forêt de Haye (fig. 2). 
Figure 2. Vue aérienne d'une voie antique (cliché Michel Loiseau avril 2000)

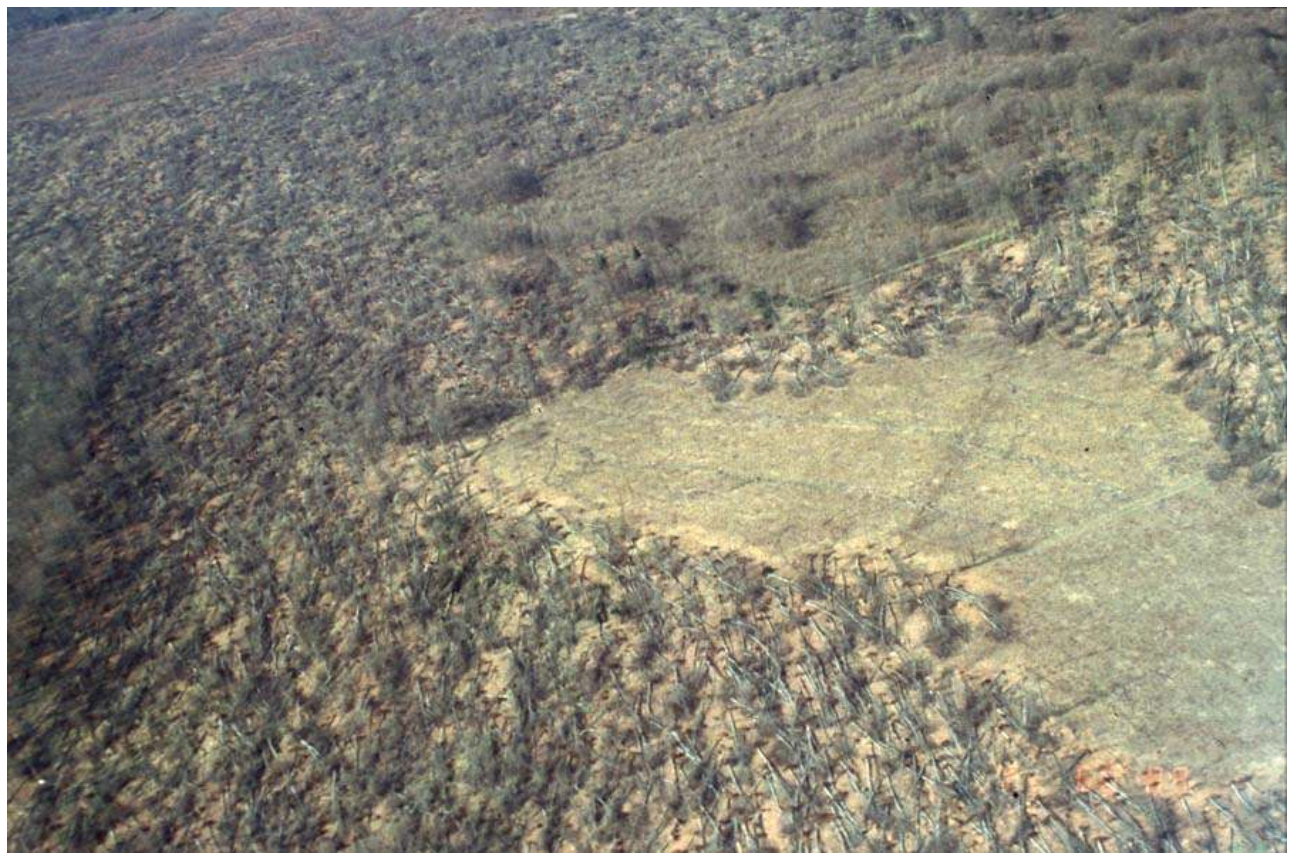

Ces contraintes et limites, qui peuvent expliquer que certaines forêts restent des déserts archéologiques, sont toutefois loin d'être rédhibitoires et sont largement compensées par le formidable potentiel des forêts.

\section{B - Une nouvelle méthode de télédétection au service de l'archéologie : le laser scanneur aéroporté}

9 Une nouvelle méthode permet de s'affranchir d'une partie de ces contraintes et peut aider au repérage et à la cartographie des sites archéologiques. Il s'agit du laser scanneur aéroporté, connu aussi sous l'acronyme anglais LiDAR. La méthode consiste en un survol à basse altitude de la zone d'étude à l'aide d'un avion équipé d'une source laser qui balaye très rapidement les surfaces au sol (Joinville et al. 2003). Les rayons laser sont reflétés par le sol ou par les objets en sursol (végétation, bâtiments). La mesure du temps mis par le signal pour revenir à l'avion permet de localiser les points avec une précision de quelques centimètres en altitude et de quelques dizaines de centimètres en planimétrie. Ce nuage de points fait ensuite l'objet d'une classification, qui consiste à séparer les points ayant atteint le sol de ceux ayant touché la végétation. Cette classification permet donc d'effacer virtuellement la forêt et fournit un modèle numérique de terrain (MNT), le plus souvent sous la forme d'une grille régulière d'altitudes, ce qui facilite les traitements ultérieurs de visualisation. Le plus immédiat consiste à réaliser un ombrage, en jouant sur l'altitude (assez rasante) et les directions d'éclairage, afin de faire apparaître l'ensemble des micro-reliefs (fig. 3).

Figure 3. Image LiDAR ombrée obtenue à partir du MNT, d'un secteur au sud de la forêt de Haye, lieudit « Champ la chèvre ».

Outre les routes et chemins forestiers, bordés de carrières, on observe les parcellaires, les voies antiques, marquées par une double ligne de murées, et les habitats composés d'enclos entourant un ou plusieurs bâtiments. 


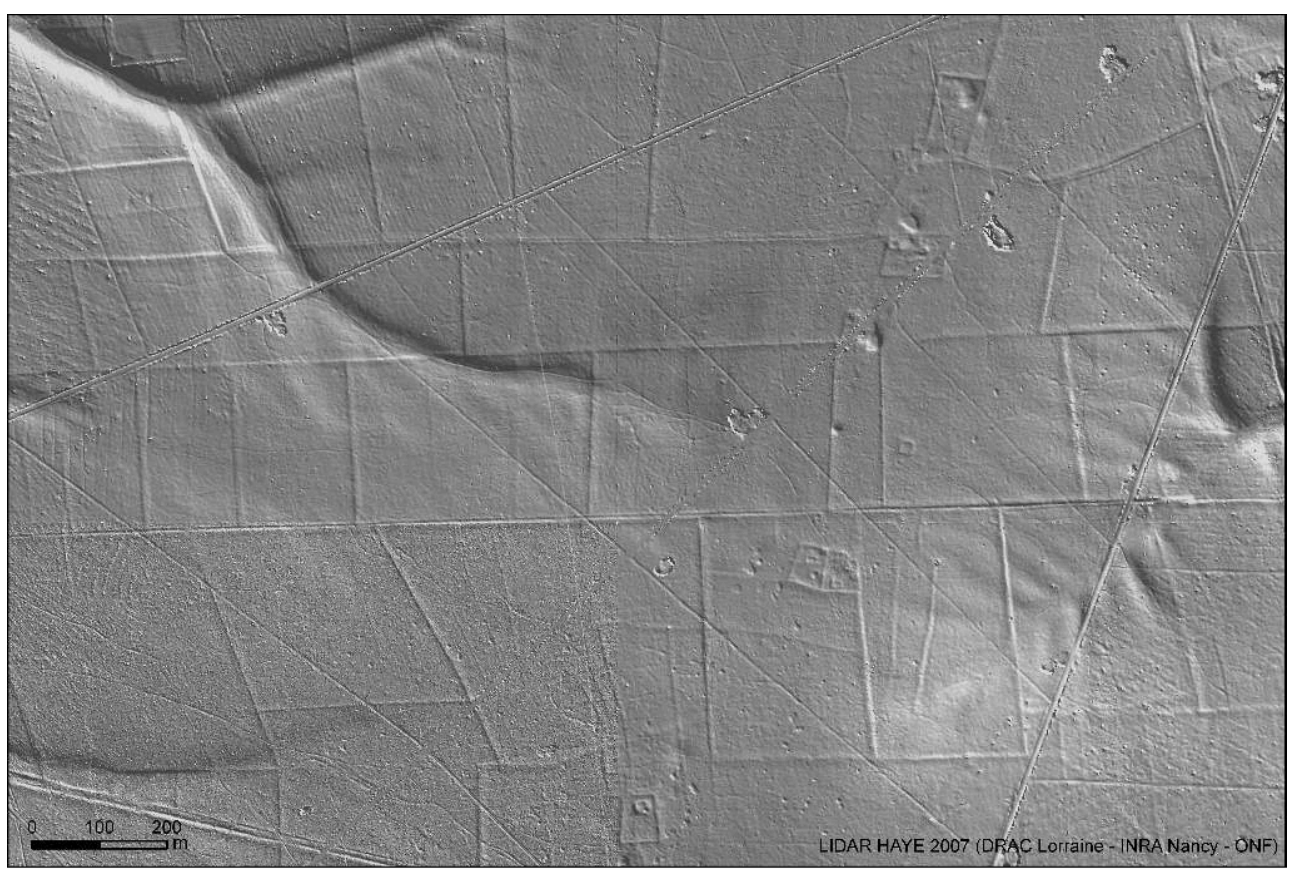

Le milieu forestier se prête particulièrement bien à cette méthode, car, on l'a vu, de nombreux vestiges y sont conservés sous forme de micro-reliefs. Cette technique de télédétection a été utilisée en milieu forestier, à des fins archéologiques, dès 2003, par une équipe du Landespflege Freiburg sur des champs bombés conservés en forêt à Rastatt, en Pays de Bade (Sittler, Hauger 2007). Des massifs forestiers ont ainsi été explorés dans divers pays, comme par exemple la forêt de Dean en Grande-Bretagne (Devereux et al. 2005), celle de Leitha Montain en Autriche (Doneus et al. 2008), dans le Michigan aux USA (Gallagher, Josephs 2008) ou à Elverum en Norvège (Risbøl et al. 2006). En France, la méthode a été utilisée pour la première fois par des archéologues durant l'hiver 2006-2007, avec notamment le vol mené au-dessus des 11600 ha du massif forestier de Haye, ce qui représente une surface tout à fait considérable.

Ce levé, réalisé en mars 2007 durant 2 jours a permis l'acquisition de 2 milliards de points, dont 250 millions ayant touché le sol et le reste ayant touché la végétation (Georges-Leroy et al. 2008). Ses objectifs étaient d'une part la réalisation de la cartographie la plus complète possible des parcellaires anciens et d'autre part l'acquisition de données sur les peuplements forestiers (hauteur des arbres notamment). Cette carte des hauteurs dominantes permet aux forestiers d'obtenir un état des lieux de la forêt et de programmer les interventions à réaliser. Mais en foresterie, la hauteur des peuplements à un âge donné est aussi un indicateur de la fertilité des sols. Un croisement entre ces données forestières, les informations archéologiques et les données écologiques devrait également permettre d'analyser l'impact de l'utilisation ancienne des sols sur l'état et le fonctionnement des écosystèmes forestiers actuels (diversité végétale, productivité des peuplements).

Pour l'archéologie, ce levé a montré que par rapport à des méthodes classiques de prospection au sol, on pouvait détecter $50 \%$ de parcellaires anciens supplémentaires. De plus la cartographie a pu être complétée dans les zones inaccessibles au sol (zones détruites par la tempête de 1999, jeunes peuplements forestiers). Ces structures apparaissent remarquablement bien. Les images LiDAR permettent en effet un recul que l'on n'a que rarement en forêt à cause de la végétation. De plus, dans le cas de 
vestiges très fugaces et très peu élevés, quasi indétectables sur le terrain, le laser scanneur a permis de multiplier pratiquement par quatre la longueur des vestiges cartographiés. Particulièrement adapté aux vestiges linéaires (voies antiques, parcellaires), le levé a aussi montré son efficacité sur des vestiges plus ponctuels : ainsi 26 habitats antiques supplémentaires ont été détectés, soit $30 \%$ du total connu et une vingtaine ont vu leur connaissance améliorée.

\section{II - La formidable richesse archéologique du massif forestier de Haye}

Figure 4 : Inventaire de quelques types de sites archéologiques recensés dans le massif forestier de Haye

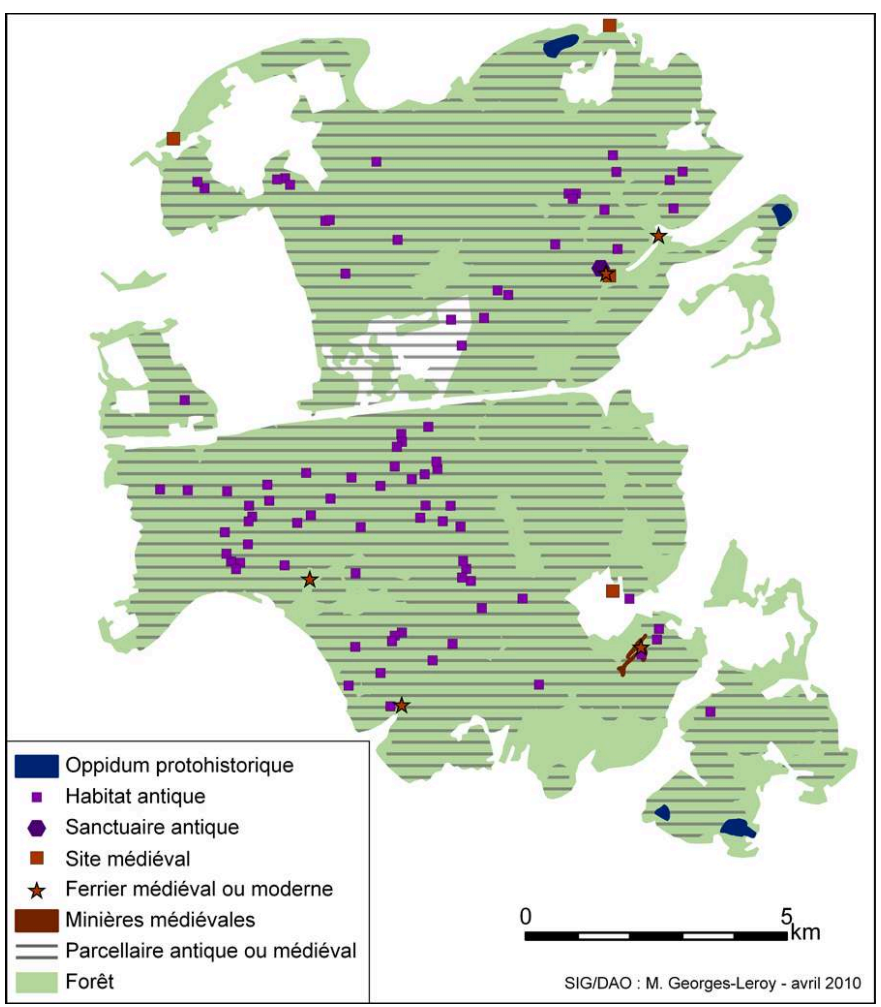

13 Le levé LiDAR et les nombreuses prospections menées depuis le XIX ${ }^{\mathrm{e}} \mathrm{s}$. par des archéologues bénévoles ${ }^{1}$ et depuis 1999 par notre équipe, ont montré que l'ensemble du massif forestier recèle des vestiges archéologiques (fig. 4). Les plus nombreux correspondent à des structures agraires et des parcellaires fossiles, associés à des fermes antiques (fig. 3). Mais on trouve aussi plusieurs sites de hauteur fortifiés de l'époque protohistorique: Camp d'Affrique à Messein, La Fourasse à Champigneulles, Pimont à Frouard et un site inédit découvert par laser scanneur dans le bois de Châtel à Chavigny. Ont également été repérés des vestiges d'extraction du minerai de fer (minette lorraine), ainsi que plusieurs ateliers de réduction de ce minerai datés des époques médiévale et moderne. De nombreux fours à chaux et carrières d'extraction du calcaire sont identifiés sur l'ensemble du massif. Le plus ancien de ces fours à chaux a été découvert à l'emplacement d'une ferme gallo-romaine et est daté des $\mathrm{XII}^{\mathrm{e}}-\mathrm{XIII}{ }^{\mathrm{e}} \mathrm{s}^{2}{ }^{2}$; les plus récents peuvent avoir fonctionné au $\mathrm{XX}^{\mathrm{e}} \mathrm{s}$. Enfin, de nombreux vestiges militaires de la fin $\mathrm{du} \mathrm{XIX}^{\mathrm{e}} \mathrm{s}$., sont dispersés dans l'ensemble de la forêt: fort de 
Frouard, batteries, mais aussi nombreux retranchements, traces de baraquements, réservoirs d'eau, etc.

Certains de ces vestiges sont associés à l'histoire de la forêt, comme les fours à chaux ou les ateliers de production du fer, mais aussi les vestiges militaires édifiés dans le cadre de la militarisation du massif forestier (Thiébaut 2004). D'autres en revanche, et ce sont les plus nombreux, témoignent au contraire d'une époque où le plateau était presque entièrement voué aux cultures et aux pâtures et non recouvert de forêt.

Il s'agit des vestiges agraires et parcellaires qui se présentent principalement sous une forme linéaire: des murées et des talus délimitant une terrasse (fig. 5). Les murées correspondent à un pierrier linéaire ne présentant pas de parement construit visible dans son état actuel, mais qui a pu en présenter un dans son état initial. Associées à des amas de pierres sèches parfois confondus avec des tumulus, ces murées témoignent d'une phase d'épierrement assez intensive, liée à la mise en culture de ces terrains calcaires aux sols peu épais. Cette pratique de l'épierrement des champs cultivés ou des prairies de fauche est attestée chez les agronomes latins ${ }^{3}$. Les talus, qui peuvent être surmontés d'une murée, sont aussi le résultat de la mise en culture de ces terrains, comme des sondages archéologiques à travers plusieurs d'entre eux l'ont montré (fig. 6). A l'origine, un mur en pierres sèches ou un pierrier informe est établi, probablement en limite de champ. Du fait d'une légère pente, ce talus a retenu les terres qui ont glissé dans la pente lors de la mise en culture et se sont accumulées contre le pierrier et l'ont même recouvert pour former ces terrasses. Ce type de vestiges a été repéré sur l'ensemble des grandes forêts implantées sur le plateau calcaire des revers de la Côte de Moselle (Georges-Leroy et al. 2003), et même plus au sud jusqu'en Côte d'Or (Mangin et al. 2000 ; Pautrat, Goguey 2007). On en trouve également sur le piémont vosgien (Heckenbenner, Meyer 2004).

Figure 5. a. Vue d'un talus (cliché M. Georges-Leroy)

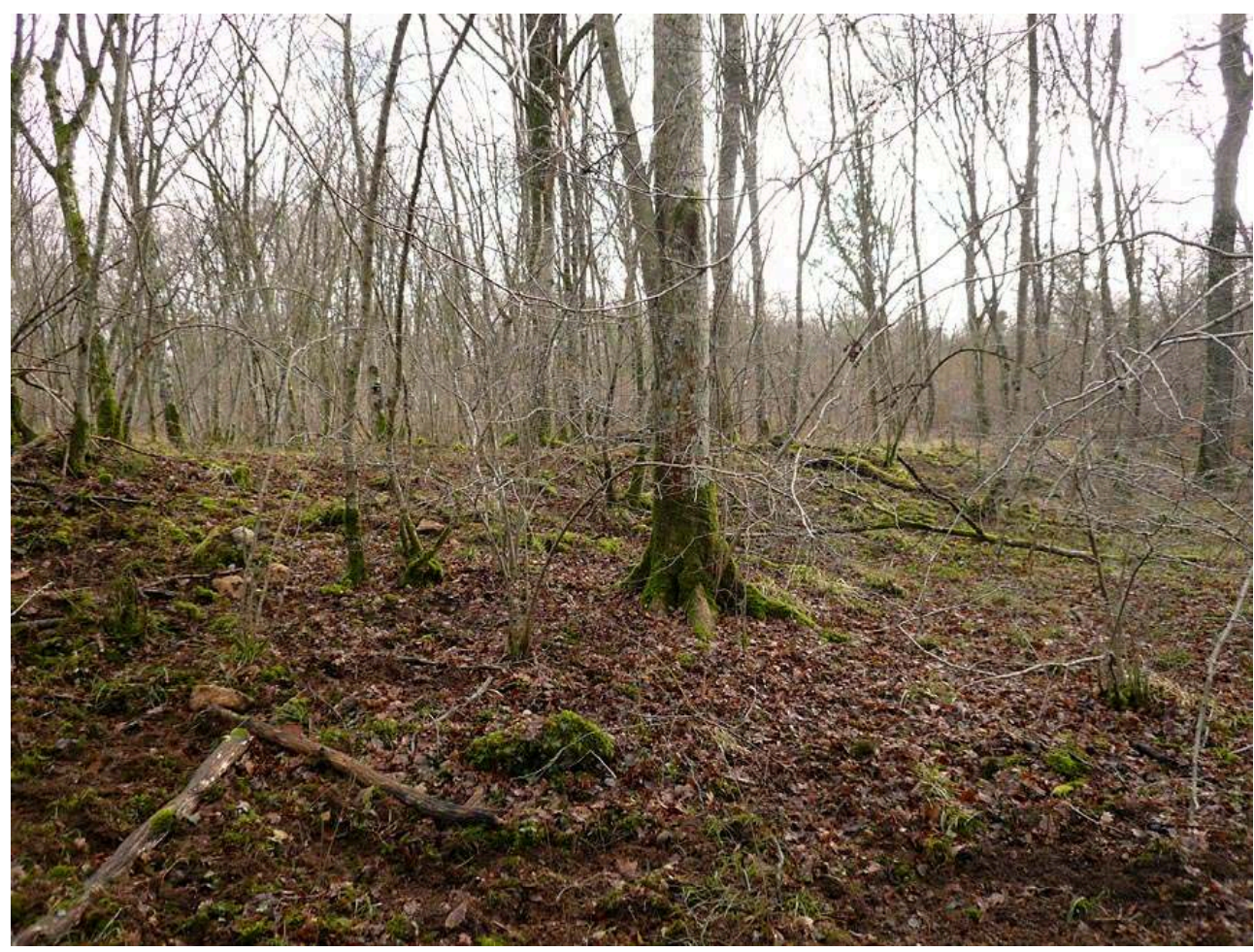


Figure 5b. Vue d'une murée (cliché M. Georges-Leroy)

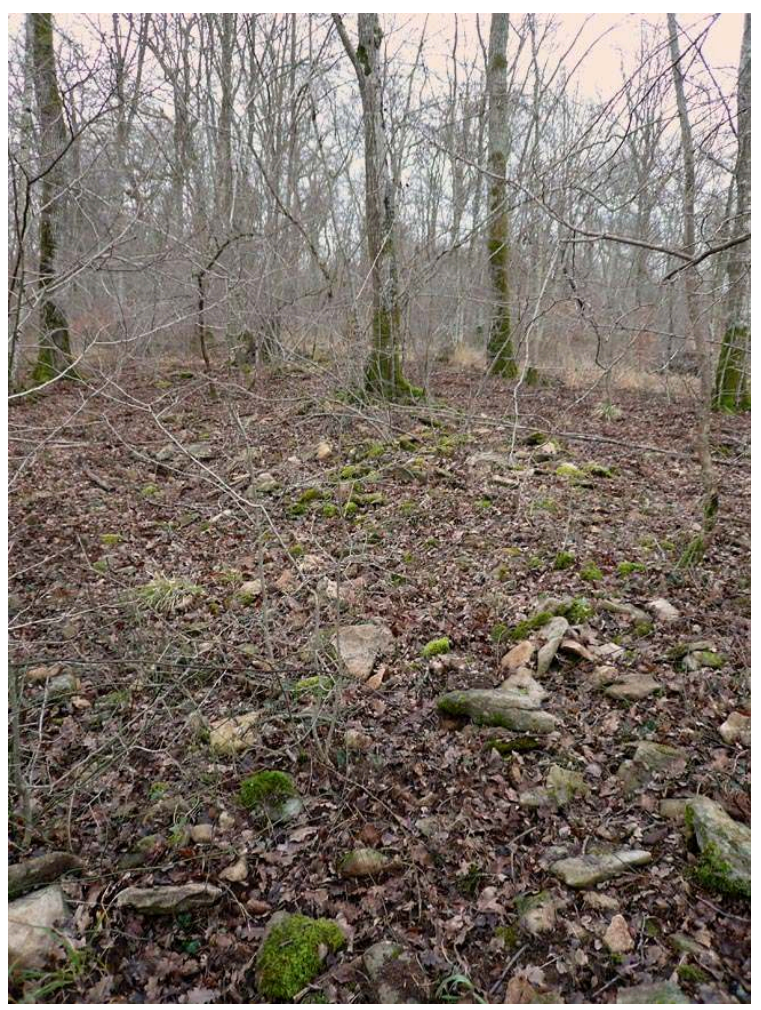

Figure 6. Vue d'un sondage réalisé à travers un talus à Maron (cliché E. Dambrine)

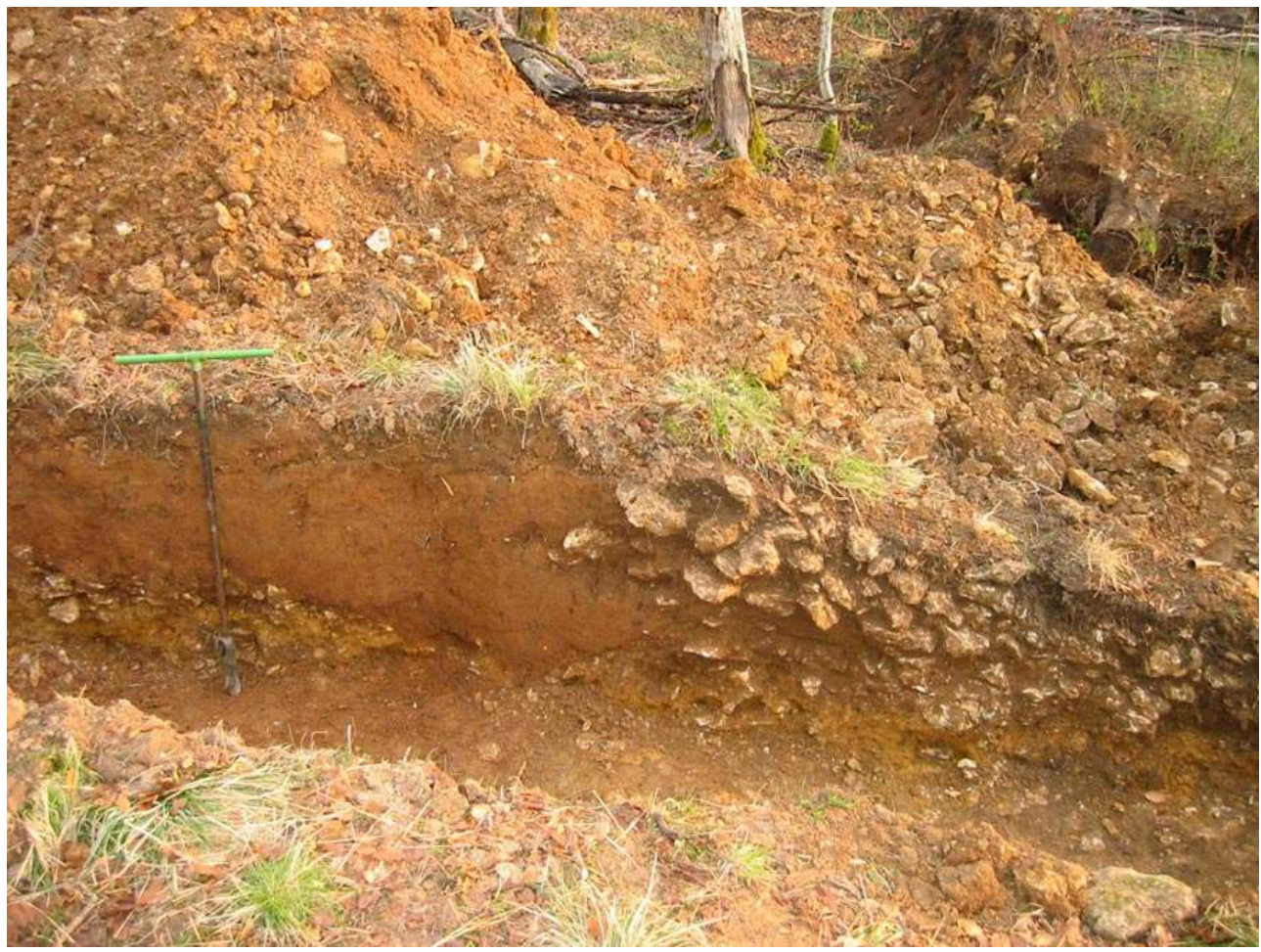

16 Une grande partie de ces murées et talus a pu être datée sans ambiguïté de l'époque antique, principalement grâce aux connexions qu'ils entretiennent avec les fermes 
antiques, mais aussi grâce à quelques sondages. Pour cette époque, ces structures agraires matérialisent de véritables limites parcellaires, en délimitant des champs mais aussi des enclos ou des voies. Les arpenteurs latins citent d'ailleurs parmi les types de limites possibles des murs de pierres, faits de pierres amoncelées sans construction, ou bien construits (Chouquer et Favory 2001, p. 188). Ces parcellaires sont desservis par un réseau de petites voies marquées par un double pierrier, elles-mêmes connectées à trois voies principales les reliant aux agglomérations du secteur: Toul, le chef-lieu de Cité des Leuques, cité dont dépendent ces parcellaires, Sion et peut-être Dieulouard (Scarpone) et Laneuveville-devant-Nancy. Ils sont aussi parsemés d'habitats se présentant sous la forme de petites fermes entourées d'un ou plusieurs enclos, de petits bâtiments isolés ou de petits habitats groupés. 85 habitats inégalement répartis dans l'espace ont été inventoriés, soit une densité de 1,1 sites $/ \mathrm{km}^{2}$ pour les seules zones où ils n'ont pas pu être masqués par les usages ultérieurs du sol. Le mobilier recueilli dans les chablis permet de les dater du début du $\mathrm{I}^{\mathrm{er}} \mathrm{s}$. à la fin du IV ou peut-être au début du $\mathrm{V}^{\mathrm{e}} \mathrm{s}$, les plus nombreux datant du milieu du $\mathrm{I}^{\mathrm{er}}$ au III ${ }^{\mathrm{e}} \mathrm{s}$. Ce mobilier atteste d'une activité agro-pastorale et la présence de déchets métallurgiques dans deux habitats implantés le long d'une grande voie témoigne aussi d'une activité de forge. Ces habitats se distinguent par leur plan et leur mode de construction (bâtiments à soubassements de pierres non liés au mortier) du modèle traditionnel de la villa romaine. Ils apportent donc un éclairage tout à fait novateur sur l'histoire des campagnes gallo-romaines, en nous permettant d'appréhender un type d'habitat, la ferme, longtemps délaissée par les archéologues au profit de la villa.

17 L'analyse des images LiDAR a aussi permis la mise en évidence de structures agraires de morphologie différente. Ces traces de culture, de type rideaux de culture, planches de labour ou billons, ressemblent fortement au résultat d'un labour réalisé à la charrue, même si les spécialistes de ces questions soulignent que l'araire utilisée de manière inclinée peut également former de telles traces agraires (Haudricourt 2000, p. 444-445). Certaines d'entre elles correspondent toutefois à des crêtes de labour, qui elles témoignent sans équivoque de l'utilisation de la charrue et donc d'une datation postérieure à l'époque gallo-romaine.

18 Grâce au laser scanneur, $600 \mathrm{~km}$ de ces vestiges agraires de toutes époques ont été cartographiés sur les 10000 ha analysés jusqu'à présent. Couvrant une surface totale de 8500 ha, on estime à 6000 ha minimum la surface couverte par les parcellaires antiques, tandis que les vestiges agraires postérieurs, qui se superposent par endroits aux parcellaires antiques, occupent une surface minimum de 2800 ha. Il s'agit là du plus grand parcellaire fossile de ce type cartographié en France et même au-delà et il présente du fait de cette surface un intérêt exceptionnel pour les archéologues. En effet, très souvent ceux-ci étudient des sites "isolés »: des habitats, des villes, des ateliers artisanaux, des nécropoles, etc., mais, hormis quelques éléments sur les voies de communication par exemple, ils n'ont que difficilement accès au paysage rural entourant ces sites. Même lors d'opérations archéologiques préventives, menées préalablement à la réalisation de grandes infrastructures comme les ZAC, ce ne sont que quelques dizaines, voire quelques centaines d'hectares d'un seul tenant qui sont étudiées. Et au total, depuis les années 1980 ce sont seulement 1150 ha très dispersés qui ont été explorés en archéologie préventive autour du massif forestier de Haye $e^{4}$, contre plus de 11000 ha d'un seul tenant en forêt. C'est une véritable planimétrie agraire fossile qui se dessine sous couvert forestier, pour peu que l'on puisse mettre en 
œuvre les moyens d'étude nécessaires. On change donc totalement d'échelle et de perspective.

\section{III - L'histoire du terroir de l'actuel massif forestier de Haye depuis 2000 ans}

\section{A - Un espace largement ouvert durant l'Antiquité}

L'histoire de ce terroir peut donc être esquissée dans ses grandes lignes, tout au moins depuis 2000 ans. En effet, si des sites protohistoriques majeurs, dont deux datés des $V^{\mathrm{e}}$ et $\mathrm{IV}^{\mathrm{e}} \mathrm{s}$. av. n. è., sont connus sur tout le pourtour du plateau dominant les Côtes de Moselle (fig. 4), on ne possède pas de renseignements sur le type d'occupation du sol ayant existé à cette époque à l'emplacement du massif forestier actuel. En effet, l'absence de découverte d'habitats protohistoriques ne prouve pas qu'il n'y en ait pas eu sur le secteur, surtout si les bâtiments étaient construits sur poteaux. Il est en tout cas assuré que le plateau limité par la boucle de la Moselle a connu, au moins dans certains secteurs, une activité agricole importante à l'Age du Fer, et tout particulièrement à la transition Hallstatt-La Tène, vers 500-400 av. n. è. En effet, sur la commune de Gondreville, à l'ouest du massif forestier, plusieurs fouilles ont montré la présence de batteries de silos de l'Age du Fer (Deffressigne et al. 2002). Et une nouvelle batterie a été fouillée en 2009 à Velaine-en-Haye (fouille S. Coubel - ANTEA). La mise en place dès l'Age du Fer de certains des parcellaires ayant fonctionné à l'époque antique ne peut donc pas être exclue en l'état des recherches.

Figure 7. Histoire du terroir de l'actuel massif forestier de Haye depuis 2000 ans

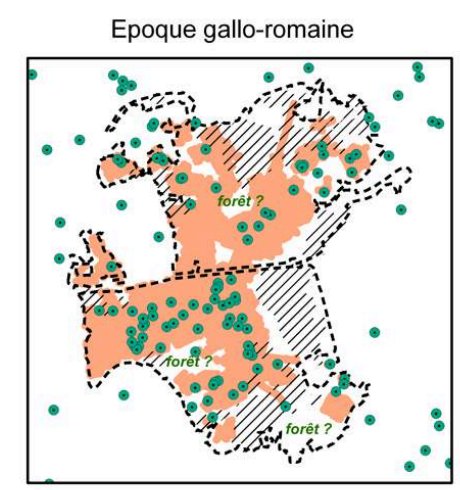

Epoques médiévales et modernes
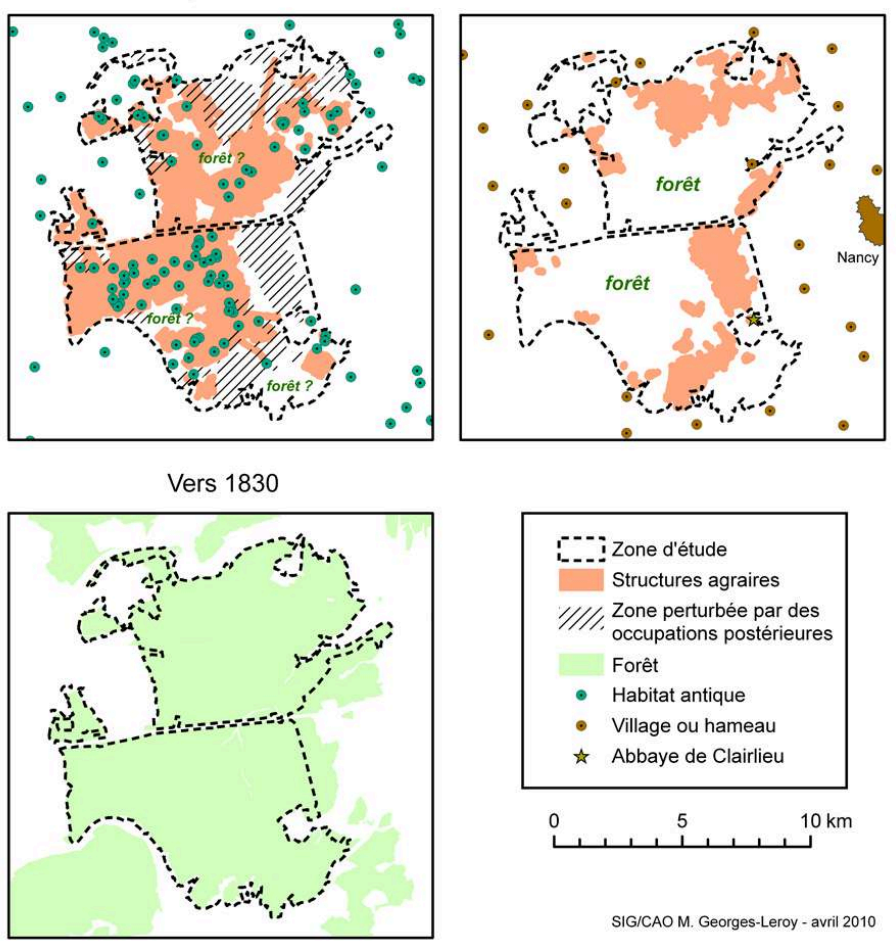
20 Pour l'Antiquité, on peut maintenant proposer de restituer un terroir largement déforesté et occupé par des champs et pâtures (fig. 7). Les structures agraires et les parcellaires antiques ont été identifiés sur une surface d'environ 6000 ha, mais il s'agit probablement d'un minimum, car elles ont peut-être été effacées dans les secteurs où ont été reconnus des vestiges agraires d'époque postérieure. L'espace où proposer, en négatif, l'existence de la forêt est donc réduit à moins de 2000 ha : probablement dans les vallons encaissés où nous n'avons quasiment jamais observé de traces de parcellaires, mais aussi sur quelques secteurs plus plats.

21 Ensuite, nous avons des interrogations concernant la reconquête forestière de cet espace, notamment à propos de sa datation. Aucun habitat postérieur à la fin du $\mathrm{IV}^{\mathrm{e}} \mathrm{ou}$ au début $d u V^{e} s$. n'a été identifié dans la forêt même, mais comme pour la Protohistoire, les habitats du haut Moyen Age sont principalement construits sur poteaux et ne laissent donc pas de traces sous forme de micro-reliefs. Les archives sont de peu de secours pour ces époques. Ainsi les premières mentions du nom de la forêt sont un peu sujettes à caution. Henri Lepage signale, sans en donner les références, qu'en 898, le roi Zwintibold donna à l'évêque de Toul une partie des bois de Heys (Lepage 1843, p. 638). Par ailleurs, le texte de 950 qui évoque la « Forestam nihilominus quae dicitus Heis ", est un pseudo-original (Arch. Départ. Moselle H151 nº 6 ; Tock 2001). Nous pensons toutefois que la reconquête forestière intervient probablement à l'extrême fin de l'époque antique ou au début de l'époque mérovingienne, car l'état de conservation des vestiges antiques suggère qu'ils ont été rapidement recouverts par la forêt pour être parvenus dans cet état jusqu'à nos jours.

\section{B - Une forêt aux limites très fluctuantes durant les époques médiévales et modernes}

22 Les archives sont plus riches pour les périodes suivantes. Nous nous basons notamment sur deux études, celle de Jean-Luc Fray pour l'époque médiévale (Fray 1986, p. 74-76) et les travaux de Nicole Guiot sur la forêt aux XVI ${ }^{\mathrm{e}}$ XVII ${ }^{\mathrm{e}}$ s. (Guiot 1959). Au Moyen Age, la forêt de Haye est répartie entre deux classes de propriétaires: les communautés d'habitants et surtout les seigneurs, qui en détiennent les deux-tiers. Parmi ceux-ci il s'agit principalement du duc de Lorraine, qui l'a reçu comme bien royal, et du comte de Chaligny. Ces archives nous donnent indirectement l'image d'un très grand massif forestier, mais il est impossible de préciser son emprise exacte. Les textes nous apportent seulement quelques indications. Ainsi en 1159, le duc Matthieu $\mathrm{I}^{\mathrm{er}}$ installe « en la vallée de Heis fort horrible et épineuse » des moines de Cîteaux qui y fondent l'abbaye de Clairlieu (Lepage 1853, p. 239). En 1225, le duc de Lorraine Mathieu II donne un secteur de la forêt de Haye à l'église de Toul. La charte en précise l'emprise, il s'agit du secteur nord du massif entre Liverdun, Pompey et le vallon Saint-Barthélémy à Champigneulles (Lepage 1843, p. 253-254).

Ce grand massif forestier a pour le moins connu des fluctuations aux marges avec la création de clairières de défrichement, mais aussi avec des reconquêtes forestières. A la fin du XVI ${ }^{e}$ s. et au début du XVII ${ }^{e}$ s., avec la pression démographique, de nombreux essarts sont accordés par les ducs aux habitants des villages limitrophes. Toutefois à cette époque, N. Guiot estime que la superficie de la forêt de Haye est sensiblement la même que de nos jours. 

des bois de la gruerie de Nancy, datée de 1613 (Arch. Départ. Meurthe-et-Moselle B 11980). Cette carte assez abîmée représente uniquement la partie ducale de la forêt, qui correspond approximativement aux deux tiers nord de la forêt domaniale actuelle et à trois bois situés à l'ouest. On possède également un plan général à l'encre de 1755 (ibid. $B$ 11800), qui correspond approximativement à la forêt domaniale actuelle. Des plans de détail en couleur, du milieu du XVIII ${ }^{\mathrm{e}}$ s., de bois appartenant au roi, à la ville de Nancy ou à des institutions religieuses (abbaye de Clairlieu, chapitre Saint-Georges) peuvent le compléter ponctuellement. Toutefois, pour une vision générale du massif forestier, il faut se référer à deux cartes moins précises : la carte en couleur des Naudin (folios 8, 1736 et 10,1733) et celle en noir et blanc de Cassini (feuilles 111, levée en 1756-1759, et 142, levée en 1754-1763). Pour le XIX ${ }^{e}$ s. nous avons surtout utilisé les minutes au 1:40 000 de la carte d'Etat-Major (feuilles 52, levée entre 1829 et 1832, et 69, levée de 1826 à 1833). Les cartes des Naudin et de l'Etat-Major ont été scannées, vectorisées et géoréférencées avant d'être superposées à la carte actuelle de l'Inventaire Forestier National.

En résumé, dès le Moyen Age, on se trouve en présence d'un important massif forestier. Les traces agraires typiques de l'utilisation de la charrue appartiennent donc à des phases de défrichement intervenues dans le courant du Moyen Age ou au tout début de l'époque Moderne (fig. 7). En effet, à l'exception de certaines traces clairement datés du $\mathrm{XIX}^{\mathrm{e}}$ s. grâce aux plans anciens, les autres sont antérieures au début du XVII ${ }^{\mathrm{s}}$ s., comme le montre l'analyse des plans anciens et le recoupement de ces vestiges par des tranchées de chasse créées à la fin du XVI ${ }^{\mathrm{e}}$ ou au début du XVII $\mathrm{s}$.

époques deux sites sont connus au cœur de la forêt, l'abbaye de Clairlieu à Villers-lès-Nancy, créée au XII ${ }^{\mathrm{e}}$ s. et démantelée à la Révolution Française, et le hameau Saint-Barthélémy à Champigneulles, dont la chapelle est attestée dès 1130 et qui est abandonné au milieu du XVII ${ }^{\mathrm{e}}$. (Lepage 1879). Une partie des parcellaires sont peutêtre à mettre en relation avec le hameau Saint-Barthélémy, voire avec l'abbaye de Clairlieu, notamment ceux implantés sur les croupes dominant le vallon reliant les deux sites. Ils peuvent remonter à l'époque médiévale, mais la culture du Val SaintBarthélémy est encore mentionnée dans des textes des XVI ${ }^{e}$ et XVII ${ }^{e}$ s. (Lepage 1879). D'autres parcellaires peuvent donc aussi dater de la phase de défrichement attestée par les textes à la fin du XVI ${ }^{\mathrm{e}}$ ou au début $\mathrm{du} \mathrm{XVII}^{\mathrm{e}} \mathrm{s}$. et qui sont le fait des communautés villageoises implantées tout autour du massif forestier.

Cet exemple d'étude archéologique forestière permet d'illustrer les fluctuations qu'a connues ce terroir. Le plateau calcaire est presque entièrement défriché et mis en culture à une époque indéterminée, mais au moins durant l'Antiquité. Puis, la forêt reconquiert probablement tout ou partie de l'espace jusqu'à nos jours, en connaissant toutefois une ou plusieurs phases de défrichement importantes dans quelques zones.

\section{IV - La mémoire des sols}

Ces occupations anciennes ont encore un impact fort sur la forêt actuelle. Les sols forestiers ont gardé la mémoire de ces occupations et la flore qui s'y développe est différente. Ce sont ces « anomalies » dans la végétation que certains archéologues ont utilisées dès les années 1970 pour détecter des sites archéologiques (Desbordes 1975). Mais elles ont aussi amené des chercheurs forestiers à s'intéresser à l'archéologie. D'où 
ce rapprochement nécessaire de disciplines différentes que notre équipe met en œuvre depuis plus d'une dizaine d'années ${ }^{5}$.

En France, comme dans d'autres pays d'Europe, les écologues distinguent classiquement deux types de forêts : les forêts récentes issues du reboisement de terres agricoles à partir du milieu du XIX ${ }^{\mathrm{e}} \mathrm{s}$. et les forêts anciennes qui étaient déjà boisées à cette époque (Dupouey et al. 2002b). Cette distinction correspond à des différences de contenu chimique des sols et surtout à des différences marquées de végétation. La portée de cette mémoire a pu être nettement reculée, grâce aux travaux menés sur deux forêts implantées sur le plateau bajocien au sud du massif forestier de Haye, les forêts de Thuilley-aux-Groseilles et de Saint-Amond. Des vestiges agraires associés à des fermes antiques y ont également été reconnus et leur impact est encore mesurable 1700 ans après leur abandon (Dupouey et al. 2002a et 2007b). Ainsi, dans le sol des secteurs $\mathrm{d}$ 'habitat et de parcelles denses, le pH et les teneurs en carbone, azote total et surtout en phosphore assimilable sont plus élevés que ceux des secteurs moins densément occupés et plus éloignés des sites d'habitat. L'enrichissement en phosphore est attribuable aux fertilisations organiques anciennes (présence de troupeaux, pratique de fumure des champs). Quant à l'élévation $\mathrm{du} \mathrm{pH}$, elle pourrait découler de la remontée et de la fragmentation des cailloutis calcaires par le labour antique. Par ailleurs, les espèces végétales nitrophiles (qui préfèrent les sites riches en azote) sont plus fréquentes sur les enclos et les sites d'habitat. Certaines espèces sont ainsi caractéristiques de ces parcellaires anciens, comme la petite pervenche (Vinca minor) ou le groseillier à maquereau (Ribes uva-crispa). Ces résultats montrent que les écosystèmes forestiers possèdent une mémoire à très long terme des transformations qu'ils ont subies (une déforestation pour mise en culture dans notre cas). Les anciens usages des terres s'expriment encore dans les propriétés des sols, dans leur fonctionnement et dans la diversité biologique.

Ces observations sont également intéressantes pour l'archéologue : d'abord utilisées pour détecter certains sites, elles peuvent également servir à les interpréter. Nous prendrons ici comme exemple les analyses de phosphore réalisées sur environ 400 ha en forêt de Haye. Ce type d'analyses (les archéologues utilisent le terme de phosphates) est parfois utilisé en archéologie dans les fouilles d'habitats pour l'interprétation des diverses structures voire sur de grandes surfaces pour identifier les habitats et les champs attenants (Zimmermann 1998). L'échantillonnage mené ici ${ }^{6}$ avait pour but la compréhension des espaces cultivés anciens et notamment la question des fertilisations. La pratique de la fumure des champs est en effet bien attestée à l'époque antique comme le laisse entrevoir la place importante consacrée au fumier chez les agronomes latins (Marbach 2004, p. 99-108). Ainsi, comme cela avait déjà été constaté à Thuilley-aux-Groseilles et Saint-Amond, les taux de phosphore les plus importants sont observables à l'emplacement et aux abords des fermes antiques. A l'exception de ces zones d'habitat, il n'a pas été possible de distinguer les espaces cultivés antiques de ceux d'époque médiévale et moderne. Pour l'époque antique, le critère d'éloignement des fermes joue un rôle fort dans les taux de phosphore : dans un secteur de parcellaire éloigné de plus de $500 \mathrm{~m}$ de tout habitat, on a mesuré les taux de phosphore les plus faibles, inférieurs même à ceux d'une zone sans parcellaire. Ces taux pourraient témoigner d'une mise en valeur de ces terres éloignées des habitats sans recours ou avec un faible recours à des amendements, sous la forme de pâturages extensifs par exemple. Toutefois cette diffusion du phosphore dans l'environnement des fermes ne 
se fait pas de façon isotrope. Ainsi dans un secteur de 210 ha, localisé sur un parcellaire quadrangulaire assez régulier, à l'est duquel 8 fermes antiques sont implantées, on voit que les taux élevés de phosphore se concentrent au niveau des fermes, mais aussi dans certaines parcelles alentours (fig. 8). Ces parcelles, dont le type de limite montre qu'elles ont à un moment au moins été mises en culture, ont donc été plus amendées que d'autres. D'ailleurs, un enclos rectangulaire de $115 \mathrm{~m}$ sur $65 \mathrm{~m}$, dans lequel aucun bâtiment n'a été repéré et qui n'a donc pas été identifié comme un habitat, présente des taux de phosphore très élevés et pourrait donc être interprété comme un enclos à bétail ou un champ intensément fumé attenant à une des fermes précédemment évoquées. On observe aussi, sur cette même figure, un taux élevé de phosphore tout le long d'une voie marquée par une double ligne de pierriers et desservant ces parcelles. C'est probablement la conséquence du passage répété de troupeaux. Le phosphore avait permis de façon analogue, de retracer les chemins suivis par les bovins, il y a plus de 3000 ans, pour revenir dans les villages de l'Age du Bronze, à l'est de l'Angleterre (Craddock 1982). Cet exemple illustre l'intérêt d'utiliser de telles méthodes sur de vastes échelles pour mieux comprendre ces espaces agraires anciens.

Figure 8. Carte interpolée des taux de phosphore à l'emplacement d'une zone de parcellaires et de fermes antiques au sud de la forêt de Haye, lieudit « Champ la chèvre »

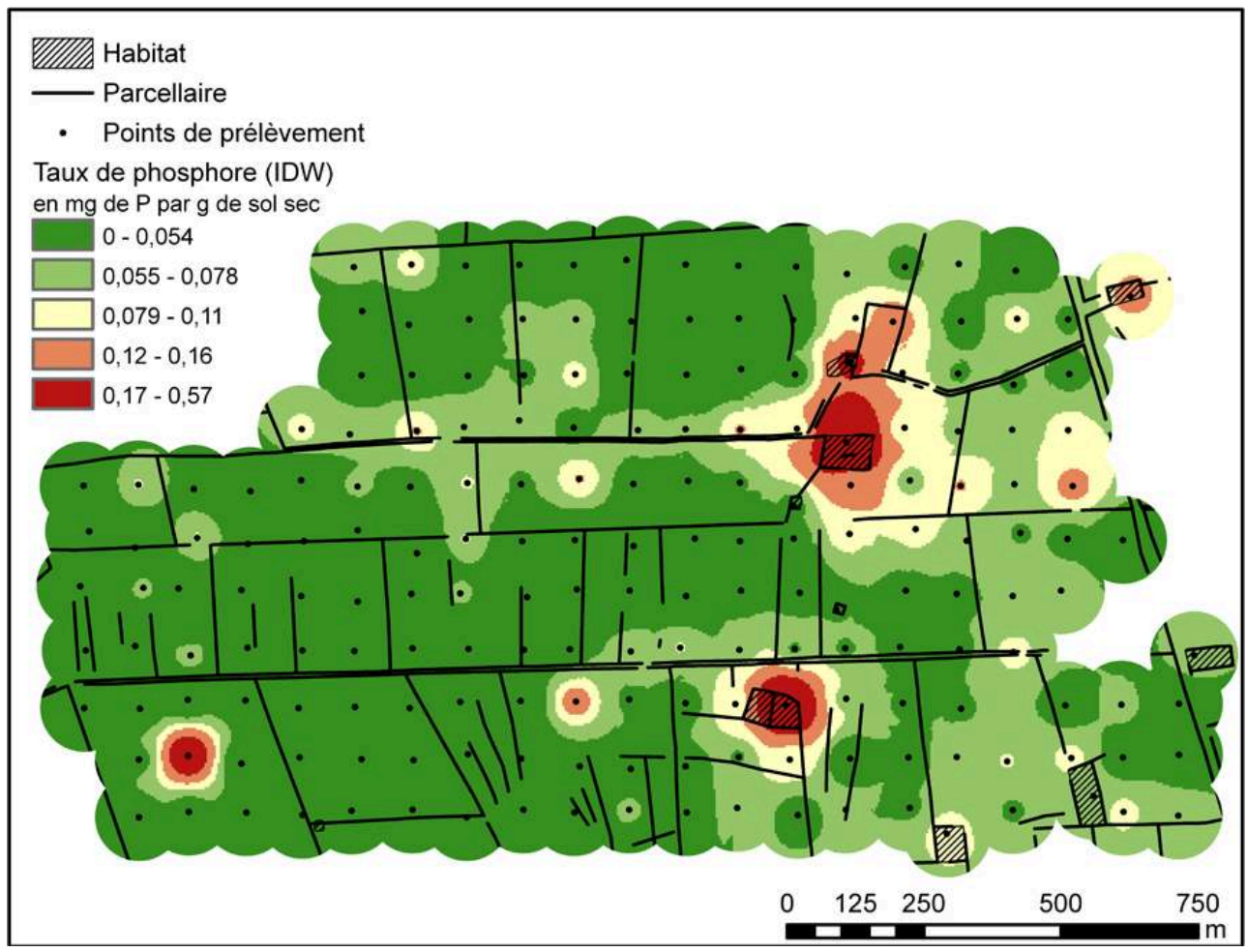

31 En conclusion, d'un point de vue pratique, le massif forestier apparaît donc comme un objet pertinent pour la recherche archéologique. Ce milieu a un fort potentiel archéologique du fait de la bonne conservation des vestiges et de la mémoire des sols forestiers.

On sait aujourd'hui que les forêts se localisent préférentiellement, dans nos paysages, sur des types de sol qui sont, en moyenne, différents de ceux des terres cultivées alentour (Badeau et al. 1999). C'est peut-être l'une des explications de la présence de ces vastes terroirs agricoles antiques dans les forêts actuelles: les agriculteurs d'hier 
auraient porté leur préférence sur des terres de nature différente de celles qu'ils choisissent aujourd'hui. Si c'est le cas, les massifs forestiers constituent un objet historique et écologique pertinent. Dans les cas où ils ont fossilisé des vestiges anciens, leur vaste surface permet d'aborder l'étude d'un terroir en changeant d'échelle et de perspective par rapport aux travaux menés en milieu ouvert. Mais la question de la représentativité de ces terroirs, aujourd'hui forestiers, pour l'étude des occupations anciennes doit cependant être posée, puisque les forêts actuelles ne sont pas situées sur les mêmes sols que le reste du territoire.

A l'heure où il est question de protéger le massif forestier de Haye de l'extension des agglomérations qui le bordent, en lui attribuant le statut de "forêt de protection ", il est intéressant de réfléchir à ces interactions entre occupations agricoles anciennes et biodiversité actuelle. Ce statut juridique défini par le Code forestier est utilisé pour des raisons liées au bien être des populations et à la protection du foncier, mais aussi pour des raisons écologiques. La composante historique nous semble donc avoir un rôle à jouer dans cette protection, tant elle influence l'occupation actuelle de l'espace et la composante écologique.

\section{BIBLIOGRAPHIE}

Badeau V., Dambrine E., Walter C. (1999). - Propriétés des sols forestiers français : Résultats du premier inventaire systématique, Etude et gestion des sols, 6, 3, p. 165-180.

BRGM (1978). - Carte géologique de la France au 1/50000. Nancy feuille 3415.

BRGM (1984). - Carte géologique de la France au 1/50000. Toul feuille 3315.

Chouquer G., Favory F. (2001). - L'arpentage romain. Histoire des textes. Droit. Techniques, Paris, Ed. Errance, $491 \mathrm{p}$.

Craddock P. (1982). - Quand les archéologues analysent les phosphates, La recherche, vol. 13, $\mathrm{n}^{\circ} 33$, p. 666-667.

Deffressigne S., Tikonoff N., Boulanger-Bouchet K., Chaussée Ch., Tesnier-Hermetey C. (2002). Les gisements d'habitat de la fin du premier âge du Fer à Gondreville - Fontenoy-sur-Moselle (54). Le stockage intensif et ses conséquences économiques et sociales, Archaelogia Mosellana, 4, Metz, p. 81-184.

Deru X., Redon B., Louvion C. et collab. (2003). - Prospections à Trépail, « Le Trou de la Baticaude » (Marne) : l'atelier de potiers et le site gallo-romain, Revue du Nord, t. 85, n 353 , p. 181-208.

Desbordes J. M. (1975). - Principes d'archéologie forestière, Revue d'Histoire et d'Art de la Brie et du Pays de Meaux, 26, p. 19-30.

Devereux B.J., Amable G.S., Crow P., Cliff A.D. (2005). - The potential of airborne lidar for detection of archaeological features under woodland canopies, Antiquity, 79, p. 648-660. 
Doneus M., Briese C., Fera M., Janner M. (2008). - Archaeological prospection of forested areas using full-waveform airborne laser scanning, Journal of Archaeological Science, p. 882-893.

Dupouey J.-L., Dambrine E., Laffite J.-D., Moares C. (2002a). - Irreversible impact of past land use on forest soils and biodiversity, Ecology, 83(11), p. 2978-2984.

Dupouey J.-L., Sciama D., Koerner W., Dambrine E., Rameau J.-C. (2002b). - La végétation des forêts anciennes, Revue Forestière Française, 54 (6), p. 521-532.

Dupouey J.-L., Dambrine E., Dardignac C., Georges-Leroy M. - éd. (2007a). - La mémoire des forêts. Actes du colloque "Forêt, Archéologie et Environnement » 14 - 16 décembre 2004, ONF - INRA - DRAC Lorraine, $294 \mathrm{p}$.

Dupouey J.-L., Sciama D., Laffite J.-D., Georges-Leroy M., Dambrine E. (2007b). - Impact des usages agricoles antiques sur la végétation en forêt de Saint-Amond : interaction avec le traitement sylvicole actuel. In : Dupouey et al. 2007a, p. 181-189.

Ferdière A. (1998). - Les prospections au sol. In : Ferdière A. - dir. - La prospection, Paris, Ed. Errance, p. 5-77. (Collection « Archéologiques »)

Fray J.-L. (1986). - Nancy-le-duc. Essor d'une résidence princière dans les deux derniers siècles du Moyen Age, Nancy, Société Thierry Alix, $344 \mathrm{p}$.

Gallagher J.M., Josephs R.L. (2008). - Using LiDAR to detect cultural resources in a forested environment: an example from Isle Royale National Park, Michigan, USA, Archaeological Prospection, 15, p. 187-206.

Georges-Leroy M., Dambrine E., Dupouey J.-L., Laffite J.-D. (2003). - Habitats gallo-romains et structures agraires fossiles du plateau de la Côte bajocienne (Meurthe-et-Moselle et Vosges). Etat de la question. In : Actualité de la recherche en histoire et archéologie agraire, Actes du colloque international AGER V, septembre 2000, Besançon, Presses Universitaires Franc-Comtoises, p. 173-180 (Annales Littéraires 764).

Georges-Leroy M., Heckenbenner D., Laffite J.-D., Meyer N., avec la collab. de Dambrine E., Dupouey J.-L. (2007). - Les parcellaires anciens fossilisés dans les forêts lorraines. In Dupouey et al. 2007a, p. 121-131.

Georges-Leroy M., Bock J., Dambrine E, Dupouey J.-L. (2008). - L'apport du laser scanneur aéroporté à l'étude des parcellaires gallo-romains du massif forestier de Haye (Meurthe-etMoselle), Bulletin de liaison AGER, 18, p. 8-11.

Guiot N. (1959). - La forêt de Haye en Lorraine aux XVI et XVII ${ }^{\mathrm{e}}$ s., Annales de l'Est, 1959-4, p. 283-312.

Gury M. (1972). - Carte pédologique du plateau de Haye, Université de Nancy et CNRS.

Haudricourt (2000). - L'homme et la charrue à travers le monde, Renaissance du livre, 617 p.

Heckenbenner D., Meyer N. (2004). - Les habitats et les parcellaires du piémont vosgien. In : Flotté P., Fuchs M. - dir. - Carte archéologique de la Gaule. La Moselle, p. 177-179.

Joinville O. de, Saur S., Bretar F. (2003). - Le levé laser aéroporté : techniques, applications et recherche, Bulletin d'information scientifique et technique de l'IGN, 74 (2003/3), p. 37-52.

Lepage H. (1843). - Le département de la Meurthe. Statistique historique et administrative. Deuxième partie, Nancy, Peiffer, 725 p.

Lepage H. (1853). -Les communes de la Meurthe. Journal historique des villes, bourgs, villages, hameaux et censes de ce département. $1^{\text {er }}$ volume, Nancy, A. Lepage imprimeur, $741 \mathrm{p}$. 
Lepage H. (1879). - Le Val Saint-Barthélemy, Mémoires de la société d'archéologie lorraine et du musée historique lorrain, 7, p. 81-129.

Mangin M., Courtadon J.-L., Fluzin Ph., de Laclos E., et collab. (2000). - Villages, forges et parcellaire aux Sources de la Seine. L'agglomération antique de Blessey-Salmaise (Côte-d'Or), Presses universitaires franc-comtoises, 517 p. (Annales littéraires de l'Université de Franche-Comté, 700 - Série « Environnement, Sociétés, Archéologie », 2).

Marbach A. (2004). - Recherches sur les instruments aratoires et le travail du sol en Gaule Belgique, Oxford, Hadrian Books Ltd, 153 p., pl. (BAR International Series 1235)

Pautrat Y., Goguey D. (2007). - Etat actuel des connaissances sur les sites archéologiques forestiers du Châtillonnais : l'exemple des parcellaires. In Dupouey et al. 2007a, p. 133-146.

Risbøl O., Gjertsen A.K., Skare (2006). - Airborne laser scanning of cultural remains in forests: some preliminary results from a Norwegian project. In Campana S., Forte M., From Space to Place. $2^{\text {nd }}$ International Conference on Remote Sensing in Archaeology. Proceedings of the $2^{\text {nd }}$ International Workshop, CNR, Rome, Italy, December 4-7, p. 107-112 (BAR International Series 1568).

Sittler B., Hauger K. (2007). - Les apports du laser aéroporté à la documentation de parcellaires anciens fossilisés par la forêt : l'exemple des champs bombés de Rastatt en Pays de Bade. In : Dupouey et al. 2007a, p. 155-161.

Thiébaut P. (2004). - La boucle de la Moselle : un enjeu stratégique (1873-1914), Etudes touloises, 111, p. 3-7.

Tock B.-M. - dir. (2001). - La diplomatique française du haut Moyen Age. Inventaire des chartes originales antérieures conservées en France, 2 vol., Turnhout, Brepols Publischers (ARTEM 4).

Zimmermann H. - trad. Nissen-Jaubert A. (1998). - La cartographie de phosphates au service de l'archéologie de l'habitat. In : Ferdière A. - dir. - La prospection, Paris, Ed. Errance, p. 207-216. (Collection « Archéologiques »)

\section{NOTES}

1. Si les recherches sur les vestiges protohistoriques ont débuté dès le XIX ${ }^{\mathrm{e}}$ s., les travaux sur les parcellaires et structures agraires n'ont été entamés qu’à partir des années 1970 par Michel Loiseau et Etienne Louis. Philippe Loué, agent de l'ONF, en a ensuite entrepris une cartographie systématique, à partir de 1995.

2. Age ${ }^{14} \mathrm{C}$ calibré : de 1161 à 1270 ap. JC (Ly-13473).

3. Par exemple chez Columelle (COL., R. R., II, 2 ; II, 17 - traduction Nisard).

4. Source : Base Patriarche du Ministère de la Culture et de la Communication - DRAC de Lorraine. La zone sur laquelle a porté le calcul est une zone de $28 \mathrm{~km}$ sur 22, localisée au niveau de la boucle de la Moselle entre Toul et Nancy. 213 opérations visant à détecter des sites archéologiques préalablement à la réalisation de travaux d'aménagement y ont été recensées de 1984 à 2009.

5. Rapprochement qui a vu un premier aboutissement lors du colloque organisé en 2004 à Velaine-en-Haye, en plein cœur de la forêt de Haye, "Mémoire des forêts. Forêt, archéologie et environnement » et qui a regroupé 200 chercheurs de divers horizons (botanistes, pédologues, archéologues, forestiers, etc.) (Dupouey et al. 2007a).

6. 350 points ont été échantillonnés en juin 2009, selon un maillage assez lâche de $100 \mathrm{~m}$ réparti sur 7 zones. Ces prélèvements et les dosages de phosphores ont été réalisés par Bruno Simon et Benoît Pollier, que nous remercions vivement. 


\section{RÉSUMÉS}

La forêt constitue un milieu avec un potentiel archéologique très fort, notamment du fait de la bonne conservation des vestiges, mais aussi de certaines propriétés chimiques des sols. En revanche, la forêt représente en elle-même un milieu très contraignant pour les études archéologiques, qui a parfois empêché ou ralenti ces études. Les nouveaux outils à la disposition des archéologues, notamment le LiDAR pour la télédétection, mais aussi les collaborations interdisciplinaires permettent aujourd'hui de réinvestir ce milieu en changeant d'échelle. Le massif forestier par sa grande surface a en effet une échelle pertinente pour aborder l'étude d'un terroir, pour peu qu'il soit assez ancien pour avoir fossilisé des vestiges antiques ou médiévaux par exemple. Ces questions sont abordées en se basant sur l'exemple du massif forestier de Haye, massif de 11600 ha localisé en périphérie de l'agglomération nancéienne, qui fait l'objet d'études interdisciplinaires depuis une dizaine d'années.

Forests are areas of high archaeological potential, particularly because of the good preservation of the remains and also because of certain chemical properties of the soil. However, forest cover is a very restraining obstacle for archaeological surveys and has sometimes hindered or even stopped them. Archaeologists now possess new tools, in particular airborne laser (LiDAR) for remote sensing but also interdisciplinary collaborative work, that enable a rediscovery of this major part of the territory, at a new scale. Forest massifs (contiguous forest areas) are a relevant scale to tackle the study of a terroir due to their large surface area, particularly if the land is old enough to have fossilized antique or medieval remains for example. Here, we develop the historical analysis of the Haye forest, situated on the outskirts of Nancy and having a total area of 11600 hectares. It has been the subject of interdisciplinary studies for the last ten years, which allowed the discovery of one of the largest Roman agricultural landscape in France.

Der Wald ist eine Umgebung mit einem sehr hohen archäologischen Potential, besonders wegen der guten Erhaltung der Überreste, aber auch aufgrund gewisser chemischer Eigenschaften des Bodens. Im Gegensatz dazu ist der Wald an sich eine sehr einschränkendes Milieu für archäologische Untersuchungen, das manchmal seine Untersuchung verhindert oder verlangsamt.

Die neuen, den Archäologen zur Verfügung stehenden Werkzeuge, vor allem LiDAR zur Fernerkundung, aber auch die interdisziplinäre Zusammenarbeit erlauben nun dieses Milieu in anderem Maßstab zu erkunden.

Der Waldkomplex durch seine große Oberfläche hat in der Tat den geeigneten Maßstab, um die Studien vor Ort anzugehen, wenn er nur alt genug ist, um zum Beispiel antike und mittelalterliche Überreste bewahrt zu haben. Diese Fragen werden am Beispiel des Waldes von Haye angegangen, einem Waldkomplex von 11600 ha in der Nähe der Stadt Nancy, der seit zehn Jahren Gegenstand interdisziplinärer Studien ist.

\section{INDEX}

Schlüsselwörter : Wald, archäologische Prospektion, Terroir, Agrarstruktur, Grundstück, Lidar, Phosphor

Keywords : forest, archaeological survey, terroir, agrarian structure, field system, lidar, phosphorus

Mots-clés : forêt, prospection archéologique, terroir, structure agraire, parcellaire, lidar, phosphore 


\section{AUTEURS}

\section{MURIELLE GEORGES-LEROY}

DRAC de Lorraine, SRA - 6, place de Chambre - 57045 Metz cedex 1, UMR 6249 Chrono-

Environnement, murielle.leroy/CHEZ/culture.gouv.fr

JÉRÔME BOCK

ONF, Pôle R\&D, DT Lorraine - Bâtiment 802 - 54840 Velaine-en-Haye

\section{JEAN-LUC DUPOUEY}

INRA, CRF - 54280 Seichamps 